OPEN

SUBJECT AREAS:

BIOCHEMICAL ASSAYS

DRUG DEVELOPMENT

PROTEOMICS

ASSAY SYSTEMS

Received

16 June 2014

Accepted

3 February 2015

Published

4 March 2015

Correspondence and requests for materials should be addressed to O.P. (poetz@nmi.de)

* Current address:

Protagen Protein

Services $\mathrm{GmbH}$, Otto-

Hahn-Str. 15, 44227

Dortmund, Germany.

$\uparrow$ These authors contributed equally to this work.

\section{Indirect protein quantification of} drug-transforming enzymes using peptide group-specific immunoaffinity enrichment and mass spectrometry

Frederik Weiß ${ }^{\dagger} \uparrow$, Anke Schnabel ${ }^{2 *} \uparrow$, Hannes Planatscher' ${ }^{1}$ Bart H. J. van den Berg' ${ }^{1}$ Bettina Serschnitzki², Andreas K. Nuessler ${ }^{3}$, Wolfgang E. Thasler ${ }^{4}$, Thomas S. Weiss ${ }^{5}$, Matthias Reuss ${ }^{6}$, Dieter Stoll7, Markus F. Templin', Thomas O. Joos ${ }^{1}$, Katrin Marcus ${ }^{2} \uparrow$ \& Oliver Poetz ${ }^{1} \dagger$

\footnotetext{
${ }^{1} \mathrm{NMI}$ Natural and Medical Sciences Institute at the University of Tübingen, Markwiesenstr. 55, Reutlingen, Germany,

${ }^{2}$ Medizinisches Proteom-Center, Ruhr-University Bochum, Bochum, Germany, ${ }^{3}$ Department of Traumatology, Eberhard Karls Universität Tübingen, Tuebingen, Germany, ${ }^{4}$ Department of Surgery, Ludwig-Maximilians-University, Munich, Germany, ${ }^{5}$ Department of Pediatrics and Juvenile Medicine, Regensburg University Hospital, Regensburg, Germany, ${ }^{6}$ Center Systems Biology, University of Stuttgart, Stuttgart, Germany, 'University of Applied Sciences, Albstadt Sigmaringen, Germany.
}

Immunoaffinity enrichment of proteotypic peptides, coupled with selected reaction monitoring, enables indirect protein quantification. However the lack of suitable antibodies limits its widespread application. We developed a method in which multi-specific antibodies are used to enrich groups of peptides, thus facilitating multiplexed quantitative protein assays. We tested this strategy in a pharmacokinetic experiment by targeting a group of homologous drug transforming proteins in human hepatocytes. Our results indicate the generic applicability of this method to any biological system.

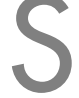
elected reaction monitoring (SRM) is a valuable tool in targeted proteomic approaches ${ }^{1}$. Proteins in biological samples are enzymatically fragmented down to peptides. Then peptides are chromatographically separated and analyzed by a triple quadrupole (QQQ) mass spectrometer. Here selected peptide precursor ions of target proteins are selectively isolated. The precursor ions are subsequently fragmented in a collision cell. Proteins are then indirectly identified and monitored by a second filter process for peptide-specific secondary ions (transitions). Indirect protein quantification is achieved by simultaneously measuring a defined, spiked amount of corresponding isotopically-labeled (C13/N15) reference peptide ${ }^{2}$. The sample consumption is low and a high number of proteins can be analyzed in parallel ${ }^{3}$. This type of mass spectrometric measurement is highly precise. However, the release of the selected proteotypic peptide during proteolysis can vary and has to be investigated target by target. Therefore, the quantification derived from the reference peptide is not absolute; however, quantification is in fact absolute when complete tryptic fragmentation without a single missed cleavage site is achieved. The use of metabolically labelled recombinant proteins will improve this in future, since a protein standard undergoes all sample processing steps ${ }^{4}$. However, the sensitivity of SRM-based protein assays is limited: this is caused e.g. by ion suppression effects that arise from the sample amount and complexity ${ }^{5}$. Peptide-specific antibody sets are used in a parallelized immunoprecipitation step to reduce sample complexity and thereby concurrent suppression effects. As a consequence sensitivity and throughput is greatly increased ${ }^{6,7}$. Although this method does not match the speed and sample throughput of other antibody-based methods, such as sandwich immunoassays ${ }^{8}$, its specificity is excellent. This highly specific protein detection is based on two independent lines of evidence: immunoaffinity capture and MS/MS readout. However, broad application of this immunoaffinity MS strategy in proteomic experiments has been restrained by the lack of suitable antibodies. The shortage of specific antibodies is still a limiting factor although rapid progress is being made in large-scale projects targeting the generation of antibodies on a proteomic-wide scale, ${ }^{9,10}$.

Recently we demonstrated that antibodies with a short C-terminal peptide epitope (triple X proteomics antibody) can be applied to enrich peptides that have a common C-terminal motif ${ }^{11}$. This strategy substantially reduces the number of antibodies required for a given set of proteins ${ }^{12}$. In the present study we combined this type 
of antibody with SRM to create a quantitative assay set-up for profiling key molecules of the hepatic drug metabolism in human which are relevant to preclinical research and drug-drug interaction profiling: the subfamily CYP3A of the human cytochrome P450 enzymes (CYP) and the ATP-binding cassette (ABC) transporter multidrugresistance protein 1 (MDR1/P-glycoprotein). Several publications have described SRM-based assays for the quantification of the cytochrome P450 enzymes (CYP) in liver, hepatocytes and hepatoma cell lines ${ }^{13-17}$. The approaches described involve laborious, time-consuming steps during the preparation of microsomes for enrichment of the low expressed CYP isoforms as required for a reliable quantification. Moreover, CYPs or other low abundant proteins can be enriched by their molecular weight, using SDS-polyacrylamide electrophoresis and quantified by LC-SRM-MS(/MS) analysis ${ }^{17,18}$. We have established a method for quantifying three homologous CYP enzymes (CYP3A4/ 43, CYP3A5, CYP3A7) and the drug transporter multidrug-resistance protein 1 (MDR1) directly from a low amounts of human hepatocyte lysates: only one TXP-antibody is required in the immunoaffinity enrichment step prior to MS-based quantification.

\section{Results}

First, we performed a C-terminal-anchored alignment of all peptide sequences derived from an in-silico tryptic digest of the CYP3A subfamily relevant for main drug transforming processes ${ }^{19}$. We identified three peptides that were suitable for SRM analysis: LQEEIDAVLPNK, EIDAVLPNK and EIDTVLPNK. Each of them carry the C-terminus LPNK. Further bioinformatic analysis revealed that a proteotypic fragment of the drug transporter MDR1 also contains this sequence and can be captured by an anti-LPNK-antibody. This multi-specific antibody (Figure 1a) was generated and purified as described previously ${ }^{11}$. We employed the synthetic peptide of each target to identify the optimal transitions to be monitored (see Supplemental Table 1 and 2 for transitions of the respective peptides). Three transitions, per peptide were defined for unequivocal peptide detection and the most intensive was selected to be the 'quantifier' transition. It should be noted, that the peptide LQEEIDAVLPNK is also present in the sequence of the enzyme CYP3A43. However, no or only very low expression in hepatocytes respectively liver was observed previously ${ }^{14,20}$.

To analyze the performance and the impact of multi-specific immunoprecipitation, we compared the results generated by means of a standard LC-SRM method with the TXP-LC-SRM method which uses the antibody specific for LPNK. We thus digested $20 \mu \mathrm{g}$ protein extract from human hepatocytes using trypsin as the fragmenting reagent. The processed sample was divided and analyzed side by side with the two different workflows. Only the more abundant enzyme, CYP3A4/43 and CYP3A5 could be unambiguously identified and quantified through the LC-SRM-based method without any enrichment step. Either no transition or only one was observed in the case of the CYP3A7- and MDR-1-specific peptides: thus, they could not be quantified. With the TXP-SRM approach, all three transitions were detectable and all four proteins were quantified (Supplemental Table 3, Figure 2, Supplemental Figure 1A-C) from the hepatocyte extract. This increase in sensitivity can be explained by the dramatic decrease of peptide complexity, which reduces ion suppression effects.

Matrix effects on the two different workflows were studied using four different amounts of hepatocyte extract (1, 5, 10 and $25 \mu \mathrm{g}$ total protein). In the TXP-SRM workflow, the signal intensity of the reference peptide was not affected by the amount of initial sample load. This demonstrates that the quality of signal and quantification is greatly improved by introducing a rapid immunopreciptitation step before LC-SRM analysis (Figure 3, Supplemental Figure 2). In contrast, in the LC-SRM workflow the increasing amounts of tryptically-fragmented hepatocyte sample resulted in decreasing signal intensity of the internal reference; whereas, the signal intensity of the endogenous peptide increased (Figure 3). This shows that the relation between the sample quantity (endogenous peptide) and the peptide standard needs to be carefully adjusted before routine quantification exclusively using LC-SRM is done. In the TXP-SRM approach it is not necessary to balance sample amount and internal standard because no signal suppression was observed. Moreover, we compared transition ion intensity ratios for each peptide since this is an additional mean for the evaluation of the specificity in peptide quantification by single reaction monitoring 5 . These ratios resulting from the endogenous and the synthetic peptide should not differ from each other. However, in some cases components of the sample influence these ratios by co-eluting molecules generating interferences in one of the monitored transitions. We observed no such interferences for any of the immunoprecipitated peptides (Supplemental Table 4). However, in the LC-SRM approach only the CYP3A4 peptide surrogate achieved acceptable values (Supplemental Table 3).

Since most antibody-based immunoassay approaches target only one molecule, our aim was to demonstrate how an antibody can capture four different analyte peptides with equal efficiency. Therefore, we mixed 1 pmol of each standard peptide (non-labelled version) with $1 \mu \mathrm{g}$ antibody and analyzed the captured fraction by eluting the peptides into the same amount of standard peptide (isotopically-labelled version). We observed for each peptide an amount of $400 \mathrm{fmol}$ of the initial peptide quantity in the eluate by forming the signal ratio of labeled-to non-labeled peptide. In sum for all four peptides the antibody's capacity was 1.6 pmol LPNK-peptide per $\mu \mathrm{g}$ antibody. The theoretical number of binding sites for one $\mu \mathrm{g}$ antibody is 13 pmol and if this number is used, the antibody's capacity is $12 \%$. More importantly, we found no evidence of preference towards one of the four peptides, which demonstrates that peptide group-specific enrichment of analytes with a shared epitope is feasible. (Supplemental Figure 3).

We also determined the technical intra- and inter-day variation. A $10 \mu \mathrm{g}$ hepatocyte protein extract from one hepatocyte donor was processed and analyzed three times on the same day for variance calculation. We calculated the inter-day variation of three independent experiments carried out on three different days. Depending on the analyte, intra-day variation ranged from 6 to $11 \%$ and inter-day variation from 9 to $19 \%$ (detailed values are given in table 1).

The lower limit of quantification (LLOQ) was determined in an analyte-free commercially available buffer preparation containing proteolytically fragmented gelatine. The LLOQ was determined according to the FDA's recommendation for bioanalytical method validation $^{21}$. Thus, the mean value for accuracy (variation) and precision (recovery) should be within $15 \%$. An LLOQ is reached when values exceed $20 \%$. Starting from $100 \mathrm{nM}$, isotopically-labeled peptides were serially diluted eight times at a ratio of $1: 3$ in digested fish gelatine, while sequence-identical endogenous peptides were kept constant at a concentration of $10 \mathrm{nM}$. Experiments were performed in triplicate. After precipitation by the anti-LPNK antibody, the peptides were measured by targeted selected ion monitoring (tSIM), using high-resolution tandem mass spectrometry (LC-MS/MS, QExactive). While the LLOQ for the MDR1 peptide was reached at $140 \mathrm{pM}$ or $1.4 \mathrm{fmol}$ absolute peptide amount, the limits for the CYP3A4/A43, Cyp3A5 and CYP3A7 were three times lower and $46 \mathrm{pM}$ or 460 amol peptide (Supplemental Figure 4).

To investigate the specificity of the antibody (on- and off-target binding), we analyzed an immunoprecipitated peptide pool, starting with $50 \mu \mathrm{g}$ hepatocyte protein extract using non-targeted liquid chromatography high resolution tandem mass spectrometry (LCMS/MS, Q Exactive). In addition to two analytes, CYP 3A4 and MDR-1 described above, the experiment revealed 8 peptides containing the targeted LPNK motif at the C-terminus (Figure $1 \mathrm{~b}$ and $\mathrm{c}$, Supplemental Table 5). The surrogate peptides for CYP3A5 and 3A7 were not detected in these analyses. We also identified 89 additional 
a

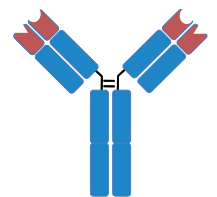

captures

Anti-LPNK
LQEEIDAVLPNK

EIDAVLPNK

EIDTVLPNK

EANIHAFIESLPNK

...LPNK

$\cdots$

\begin{tabular}{|c|c|c|c|}
\hline b-terminus & $\begin{array}{c}\text { observed } \\
\text { peptides }\end{array}$ & $\begin{array}{c}\text { theoretical } \\
\text { peptides }\end{array}$ & $\begin{array}{c}\text { ratio } \\
{[\%]}\end{array}$ \\
\hline LPNK & 10 & 70 & 14 \\
\hline LWNK & 5 & 35 & 14 \\
\hline LINK & 10 & 129 & 8 \\
\hline LYNK & 5 & 67 & 7 \\
\hline LVNK & 7 & 102 & 7 \\
\hline IPNK & 3 & 44 & 7 \\
\hline VPNK & 3 & 49 & 6 \\
\hline LTNK & 5 & 93 & 5 \\
\hline LANK & 6 & 112 & 5 \\
\hline LFNK & 4 & 81 & 5 \\
\hline LGNK & 6 & 126 & 5 \\
\hline LLNK & 9 & 195 & 5 \\
\hline LMNK & 2 & 44 & 5 \\
\hline FANK & 2 & 50 & 4 \\
\hline IFNK & 2 & 54 & 4 \\
\hline LNNK & 3 & 88 & 3 \\
\hline LSNK & 4 & 124 & 3 \\
\hline LENK & 5 & 165 & 3 \\
\hline LPGK & 5 & 177 & 3 \\
\hline LPNR & 2 & 73 & 3 \\
\hline LDNK & 2 & 75 & 3 \\
\hline LQNK & 3 & 128 & 2 \\
\hline ILNK & 2 & 103 & 2 \\
\hline & & & \\
\hline
\end{tabular}

Figure $\mathbf{1} \mid$ (a) Peptide group-specific antibodies. TXP antibodies target peptide subsets of global tryptic digests that share the same terminus.

(b) Frequency of C-termini in a peptide pool immunoprecipitated by the antibody anti-LPNK. Peptides were immunoprecipitated from $50 \mu \mathrm{g}$ tryptically digested hepatocyte and analyzed by high resolution tandem-mass spectrometry. Theoretical number of peptides harboring the respective C-termini were calculated from an in silico digest of the Uniprot database (UniProt Homo Sapiens Reference Proteome Set database (2014 Release 1)). (c) Amino acid frequency in the binding motif of the antibody, calculated based on the high resolution tandem-mass spectrometry analysis.

peptides with different motifs for one amino acid and six peptides differing in two amino acids. This mainly affected leucine and proline. Our findings provide evidence for a strong preference for LPNK-motif binding (Figure 1b).

We applied this multiplex assay in a pharmacokinetic experiment. Primary hepatocytes were treated over a three-day period with two different statins, pravastatin and atorvastatin. Cells were harvested after 6, 12, 24, 48, and 72 hours and analysed with the established TXP-SRM assay (Figure 4). Atorvastatin is known to induce CYP3A4 via the nuclear pregnane $\mathrm{X}$ receptor $(\mathrm{PXR})^{22}$. CYP3A4 expression is not influenced by pravastatin treatment, as Feidt et al. described ${ }^{22}$. The impact of atorvastatin and pravastatin on the protein expression of CYP3A5, CYP3A7 and MDR-1 has not been described so far. In our experiment we observed no induction of the monitored enzymes and the transporter in the pravastatin-treated cells, as compared to the solvent control (DMSO) (Figure 4a-d). We observed three-fold and ten-fold induction of CYP3A4 (Figure 4a) and 3A7 (Figure 4c) by atorvastatin, with a peak after 24 h however, CYP3A5 was not affected (Figure 4b). A late two- to three-fold induction by atorvastatin was observed in MDR-1 protein expression over time (Figure 4d). Our results confirm prior findings relating to mRNA expression and enzymatic activity analyses for CYP3A4 induction by atorvastatin. To our knowledge, we are the first to report an increase in CYP3A7 and MDR-1 protein levels after atorvastatin treatment (Figure $4 \mathrm{c}$ and $\mathrm{d}$ ). The induction mechanism most likely involves the PXR mediated transcriptional regulation because functional PXR binding sites in the $5^{\prime}$-flanking region of both genes were identified previously ${ }^{23,24}$.

\section{Discussion}

We established a new multiplex MS-based immunoassay (TXPSRM) using one antibody. The antibody's specificity towards the short C-terminal peptide sequence LPNK allows the quantification of three drug metabolizing enzymes and one drug transporter from a minute amount of sample. Through an analysis of statin-treated human hepatocytes, we indicated the relevance of this method to pharmacokinetic investigations. By introducing a rapid immunoaffinity step into the LC-SRM-workflow sensitivity was improved more than ten-fold and a direct analysis of the transporter and the three drug-metabolizing enzymes without membrane fractionation or microsome preparation facilitated. In typical induction experiments the cytochrome isoforms 1A2, 2B6, 2C8, 2C9, 2C19, and $2 \mathrm{E} 1$ are investigated in addition to CYP $3 \mathrm{~A} 4$ by mRNA expression analyses and/or activity assays ${ }^{25}$. Future immunizations targeting the termini FSGR (2B6, 2C8, and 2E1), LAER (2C9 and 2C19) and VIGR (1A2) will allow assay development for those enzymes. These assays might allow the monitoring of induction kinetics of a larger cytochrome P450 set on protein level, because problems caused by enzyme inhibition or mRNA degradation are avoided.

Apart from the analysis of homologue protein families, the strength of this approach lies in its generic applicability to any biological system as it offers the advantage of using a small number of antibodies for the quantification of a large number of proteins.

\section{Methods}

Isolation and cultivation of primary human hepatocytes. Primary hepatocytes were isolated and cultured as described previously ${ }^{26}$. Preparations from human liver resections from patients undergoing partial hepatectomy were performed according to the guidelines of the charitable state-controlled foundation HTCR (Human Tissue and Cell Research) Regensburg, Germany and the institutional guidelines for liver resections of tumor patients with primary or secondary liver tumors, Technical University Munich, MRI, Munich, Germany. The scientific use of human hepatocytes was approved by the local ethics committees of the Ludwig-Maximilians-University of Munich $^{27}$ and the Technical University Munich, MRI, Munich ${ }^{28}$, Germany. Written informed consent was obtained from all patients.

Hepatocytes were plated at a density of $1.5 * 10^{6}$ cells/well on collagen-coated 6well plates. Cells were allowed to settle down to the collagen layer. Culture media was replaced by serum-free Williams Medium E (Pan-Biotech-GmbH), supplemented with albumin $(0.1 \%(\mathrm{v} / \mathrm{v}))$, after transport. Before the induction experiment was 
TXP-SRM
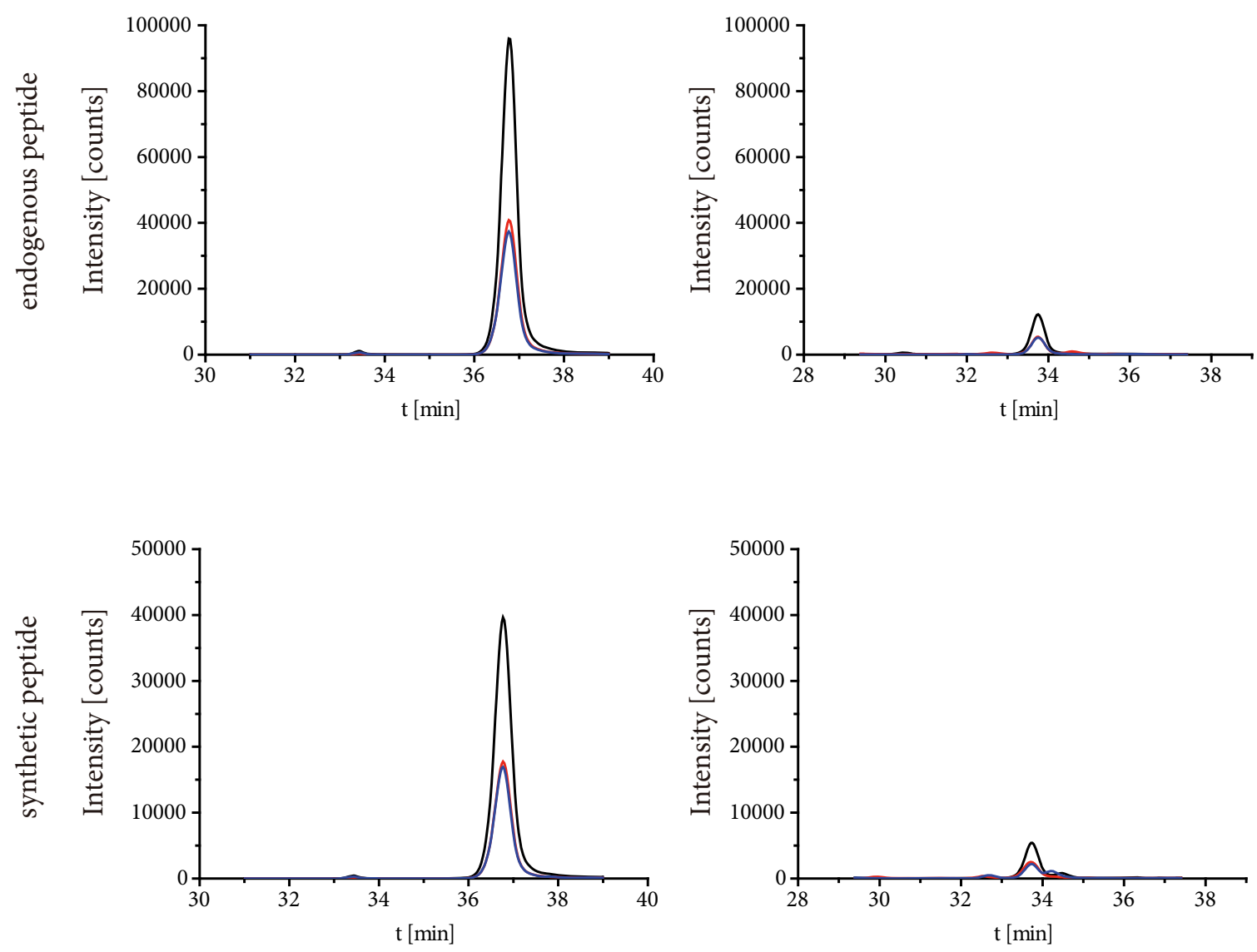

LC-SRM

$\mathrm{t}[\mathrm{min}]$

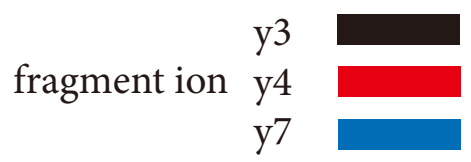
hepatocyte protein extract was digested and spiked with $100 \mathrm{fmol}$ synthetic standard. The samples were either analyzed by TXP-SRM or LC-SRM workflow. Ion chromatograms of the peptide fragments y3, y4 and y7 are shown.

started, cells were starved for $24 \mathrm{~h}$ at $37^{\circ} \mathrm{C}$ in a humidified chamber with $95 \% / 5 \%$ air/ $\mathrm{CO}_{2}$, penicillin/streptomycin $(100 \mathrm{U} / \mathrm{mL})$, stabilized L-glutamine $(2 \mathrm{mM})$, dexamethasone dihydrogenphosphate $(0.025 \%(\mathrm{v} / \mathrm{v}))$ and ITS-X (5 mg insulin, $3.35 \mu \mathrm{g}$ sodium-selenite, $2.75 \mathrm{mg}$ transferrin and $1 \mathrm{mg}$ ethanolamine)(Invitrogen), further referred to as SFM.

Cells were treated with $60 \mu \mathrm{M}$ atorvastatin $30 \mu \mathrm{M}$ pravastatin and solvent control (DMSO) in SFM for 6, 12, 24, 48, and 72 hours. Cells were harvested in PBS, supplemented with protease inhibitors (Roche, Complete Mini EDTA-free). Cell suspensions were spun down for $5 \mathrm{~min}$ at $500 \mathrm{rcf}$ at $4^{\circ} \mathrm{C}$ and washed once in $150 \mu \mathrm{L}$ NaPP-buffer (0.1 M, pH 7.4), containing $250 \mathrm{mM}$ sucrose and protease inhibitors (Complete Mini EDTA-free, Roche). Cells were homogenized by ultra-sonification and lyophilized. Total protein content was determined by amino acid analysis on an HPLC system (2695, Waters) using the 6-aminoquinolyl-N-hydroxysccinimidyl carbamate (AQC) reagent and calculated as described previously ${ }^{29,30}$.

Peptide Standards. Peptide standards (Thermo Fisher) were quantified by amino acid analysis on an HPLC system (2695, Waters) using the AccQ Tag derivatisation protocol (Waters) as described previously ${ }^{29,30}$.

Protein Digestion. Proteins were precipitated by adding acetone to $80 \% \mathrm{v} / \mathrm{v}$ and were incubated overnight at $-80^{\circ} \mathrm{C}$. Samples were centrifuged at $10.000 \mathrm{rcf}$ at $4{ }^{\circ} \mathrm{C}$ for $5 \mathrm{~min}$. Supernatant was discarded and proteins were air-dried for $5 \mathrm{~min}$. An amount of $100 \mathrm{fmol}$ per internal standard peptide was added after resolving the proteins in $8 \mathrm{M}$ urea, $0.05 \%$ 3-((1-(Furan-2-yl) undecyloxy) carbonylamino) propane-1sulfonate (ProteaseMAX, Promega) in $50 \mathrm{mM}$ ammonium bicarbonate $\mathrm{pH}$ 7.4. Proteins were reduced with $5 \mathrm{mM}$ dithiothreitol (DTT) in the presence of $0.05 \%$
ProteaseMAX (Promega) for $20 \mathrm{~min}$ at $56^{\circ} \mathrm{C}$ and alkylated with $15 \mathrm{mM}$ iodacetamide (IAA) in $50 \mathrm{mM}$ ammonium bicarbonate for $15 \mathrm{~min}$ at RT in the dark. Finally samples were digested with trypsin using an enzyme:substrate ratio of $1: 10$ at $42^{\circ} \mathrm{C}$ for $4 \mathrm{~h}$ on a shaker. Enzymatic reaction was terminated by boiling for $5 \mathrm{~min}$.

Immunoprecipitation. Enzymatically fragmented proteins were mixed with $2 \mu \mathrm{g}$ anti-LPNK antibody and incubated at $500 \mathrm{rpm}$ at $30^{\circ} \mathrm{C}$ for $1 \mathrm{~h}$ on a shaker (Eppendorf). Peptide-antibody complexes were precipitated by adding $10 \mu \mathrm{L}$ protein G-coated magnetic microspheres (Invitrogen). Beads were washed using disposable $200 \mu \mathrm{L}$ pipette tips and a ring magnet. Five wash cycles were performed with $100 \mu \mathrm{L}$ PBS, supplemented with $0.3 \% \mathrm{n}$-octyl- $\beta$-glucoside (SIGMA Aldrich) followed by a final wash using PBS only. Peptides were eluted by applying $20 \mu \mathrm{L} 1 \%$ formic acid.

Peptide desalting. C18 material containing tips (OMIX, Agilent) were preconditioned 5 times with $100 \mu \mathrm{L} 50 \%$ acetonitrile and 5 times with $100 \mu \mathrm{L} 0.1 \%$ trifluoric acid (TFA). Peptides were extracted from the sample by pipetting $100 \mu \mathrm{L} 8$ times. Peptide elution was carried out in two steps using $15 \mu \mathrm{L} 55 \%$ acetonitrile, $0.1 \%$ TFA. The peptide solutions were dried in a vacuum centrifuge and re-solubilized in $25 \mu \mathrm{L} 0.1 \%$ TFA, $1 \%$ acetonitrile.

LC-SRM. Peptide quantification was performed on a hybrid triple quadrupole ion trap mass spectrometer (4000QTrap; ABISciex) equipped with a nanoelectrospray ion source. Prior MS-analysis desalted or immunoprecipitated peptide samples were separated on a nanoliter flow HPLC system (Ultimate, Dionex) using two precolumn as described previously ${ }^{31}$. After injection $(15 \mu \mathrm{L})$, peptides were trapped and desalted on a pre-column $\left(0.3 \mathrm{~mm}\right.$ I.D. $\times 5 \mathrm{~mm}$ PepMap ${ }^{\mathrm{TM}}$, Dionex $)$ at a flow rate of $30 \mu \mathrm{L} /$ 


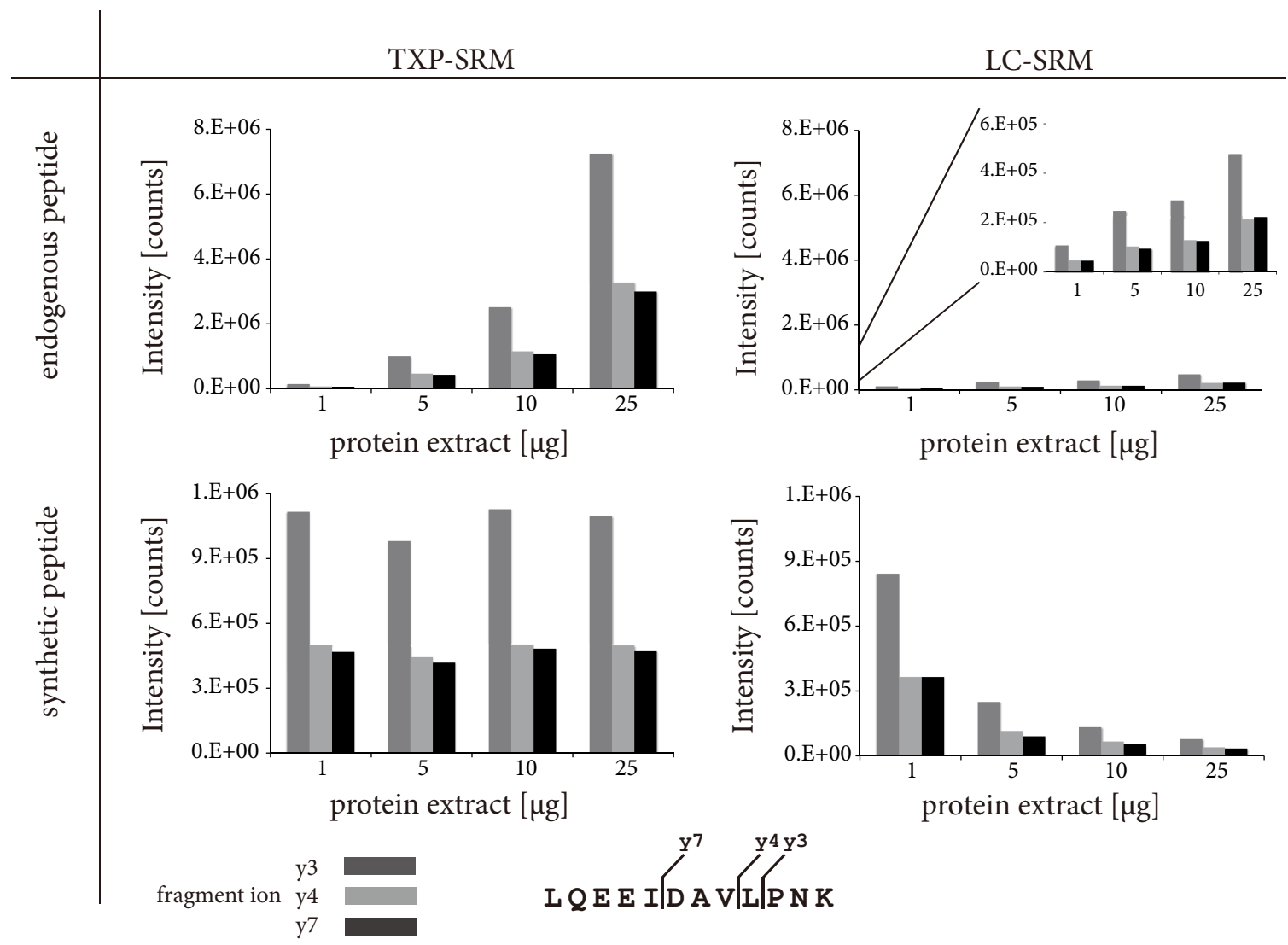

Figure 3 Comparison of integrated signals of endogenous cytochrome P450 3A4 peptide and synthetic standard. One, five, ten or $25 \mu \mathrm{g}$ hepatocyte protein extract was digested and spiked with $100 \mathrm{fmol}$ synthetic standard. The samples were either analyzed by TXP-SRM or LC-SRM workflow. Integrated signals of the peptide fragments $\mathrm{y} 3, \mathrm{y} 4$ and $\mathrm{y} 7$ are plotted against the analyzed amount of protein extract.

min in $0.1 \%$ TFA for 6 min. Peptides were transferred to the separation column $\left(75 \mu \mathrm{m}\right.$ I.D. $\times 250 \mathrm{~mm}$ PepMap ${ }^{\mathrm{TM}}$ column, Dionex $)$ and separated by applying a linear gradient of mobile phase (A: $0.1 \%$ formic acid, B: $84 \%$ acetonitrile $/ 0.1 \%$ formic acid) from $5 \%$ B to $40 \%$ B over a period of $35 \mathrm{~min}$ with a flow of $300 \mathrm{~nL} / \mathrm{min}$.

The mass spectrometer was run in multiple-reaction monitoring mode. Peptidespecific tuned declustering potential (DP), entrance potential (EP), collision cell energy (CE), collision cell exit potential (CXP), and retention times are given in supplemental table 2 . Three transitions per surrogate peptide were monitored. The scheduled option was employed for all data acquisition with a target scan time of $3 \mathrm{~s}$, cycle time of $30 \mathrm{~min}$ and an $8 \mathrm{~min}$ detection window. Data was processed using MultiQuant TM 3.0 (ABSciex) applying the MQL algorithm for peak integration. Retention time window was set to $4 \mathrm{~min}$ and a three-point smooth with a peak splitting factor of 2 applied. No noise subtraction was done. The endogenous amounts of CYP3A4, CYP 3A5, CYP3A7 and MDR1 were calculated from integrated areas of the respective ion-chromatogram of the surrogate peptide transitions. Briefly, the average of the three peak area ratios were formed: internal standard peptide transitions: endogenous peptide transitions.

Epitope analysis by LC-MS. To investigate the specificity of the anti-LPNK antibody LC-Full-MS analyses of three immunoprecipitation replicates from digested hepatocyte lysates were performed. We incubated $5 \mu \mathrm{g}$ anti-LPNK antibody with $50 \mu \mathrm{g}$ tryptically digested hepatocyte lysate. Peptide antibody complexes were precipitated with $25 \mu \mathrm{L}$ protein G-coated magnetic microspheres (Invitrogen). Peptides were eluted in $20 \mu \mathrm{L} 1 \%$ formic acid. The entire eluate $(20 \mu \mathrm{L})$ was injected and separated on a nanoliter flow HPLC system (U3000, Dionex). Peptides were loaded on an Acclaim PepMap100 C18 $\mu$-pre-column $(0.3 \mathrm{~mm}$ I.D. $\times 5 \mathrm{~mm}, 5 \mu \mathrm{m}$ particles, Dionex) for $3 \mathrm{~min}$ at a flow rate of $20 \mu \mathrm{L} / \mathrm{min}$ in $0.1 \%$ TFA. The peptides were separated for $20 \mathrm{~min}$ by an Acclaim PepMap RSLC C18 $(75 \mu \mathrm{m}$ I.D. $\times 150 \mathrm{~mm}$, $2 \mu \mathrm{m}$ particles, Dionex) using a linear gradient from $5 \%$ to $55 \% \mathrm{~B}$ at a flow rate of $300 \mathrm{nl} / \mathrm{min}$ at $40^{\circ} \mathrm{C}$. A Full-MS analysis was performed on a QExactive ${ }^{\mathrm{TM}}$ Plus

Table 1 | Intra- and inter-assay variances of the TXP-SRM assays targeting cytochrome P450 3A4, 3A5, 3A7, and multi-drug resistance protein 1

\begin{tabular}{|c|c|c|c|c|c|}
\hline & \multicolumn{3}{|c|}{ Replicate [fmol $\mu \mathrm{g}^{-1}$ ] } & \multirow[b]{2}{*}{ Mean $(\mathrm{n}=3)$} & \multirow[b]{2}{*}{ Intra day variation [\%] } \\
\hline & 1 & 2 & 3 & & \\
\hline CYP 3A4 & 13.0 & 15.1 & 15.3 & 14.5 & 9.0 \\
\hline CYP 3 A5 & 0.7 & 0.8 & 0.8 & 0.7 & 7.6 \\
\hline CYP 3A7 & 0.3 & 0.3 & 0.3 & 0.3 & 10.2 \\
\hline \multirow[t]{3}{*}{ MDR-1 } & 1.1 & 1.2 & 1.2 & 1.2 & 6.9 \\
\hline & \multicolumn{3}{|c|}{ Replicate [fmol $\left.\mu \mathrm{g}^{-1}\right]$} & & \\
\hline & 1 & 2 & 3 & Mean $(n=3)$ & Inter day variation [\%] \\
\hline CYP 3A4 & 22.6 & 18.7 & 15.3 & 18.9 & 19.3 \\
\hline CYP $3 A 5$ & 1.0 & 0.9 & 0.8 & 0.9 & 11.2 \\
\hline CYP 3A7 & 0.4 & 0.4 & 0.3 & 0.4 & 13.2 \\
\hline MDR-1 & 1.0 & 1.0 & 1.2 & 1.1 & 8.9 \\
\hline
\end{tabular}


a

$$
\begin{gathered}
\text { Cytochrome P450 3A4/43 } \\
\text { LQEEIDAVLPNK }
\end{gathered}
$$

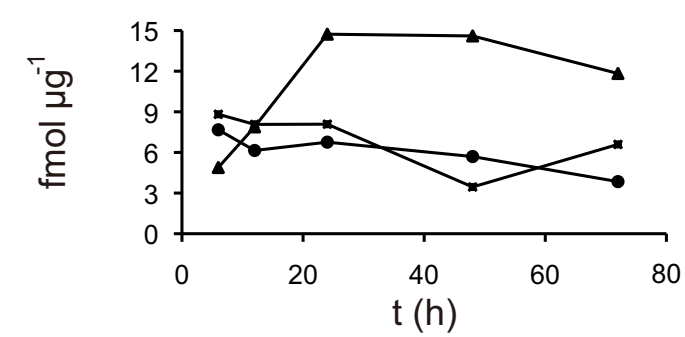

\section{Cytochrome P450 3A7 \\ EIDTVLPNK}

c

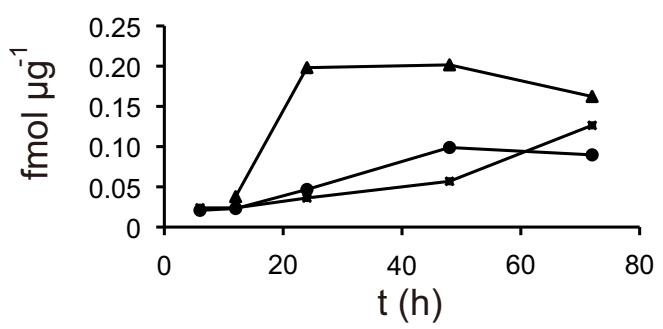

b

Cytochrome P450 3A5
EIDAVLPNK

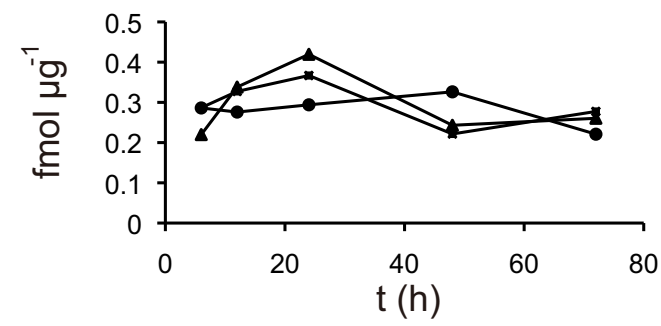

Multidrug resistance protein 1 EANIHAFIESLPNK

d

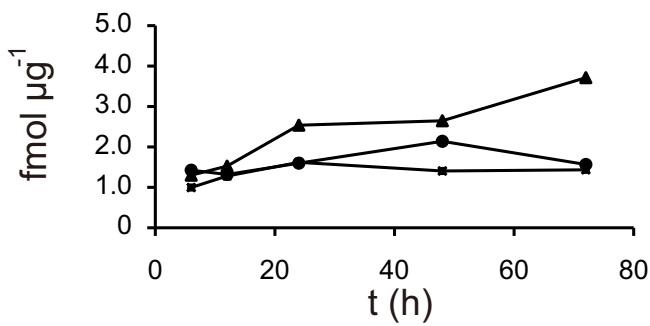

Figure 4 Induction of cytochrome P450 3A4, 3A5, 3A7, and multi-drug resistance protein 1. Human hepatoycte cultures (6-well format, 3 days after isolation) were treated with $60 \mu \mathrm{M}$ atorvastatin (triangle), $30 \mu \mathrm{M}$ pravastatin (circle) and solvent control (cross, DMSO) for 6, 12, 24, 48, and 72 hours. Cells were harvested and homogenized. $10 \mu \mathrm{g}$ protein extract of each time point was analyzed using the TXP-SRM assays for cytochrome P450 (a) $3 \mathrm{~A} 4$, (b) 3A5, (c) 3A7, and (d) multi-drug resistance protein 1.

Orbitrap Mass Spectrometer (Themo Scientific). In MS mode the resolution was set to 70.000 , the scan range from $300-2000 \mathrm{~m} / z$, the AGC-target limited to $3 \mathrm{e} 6$, and the injection time to $100 \mathrm{~min}$. For MS2 mode a top 10 method was selected, the precursor AGC-target limited to 5e5, the maximum injection time set to $60 \mathrm{~min}$, and the normalized collision energy adjusted to a value of $25 \%$. No underfill ratio was chosen and only $2+, 3+$, and $4+$ charged ions included. The dynamic exclusion time was set to 5.0 s. For identification both Mascot- and SEQUEST-algorithm were chosen for searching in the UniProt Homo Sapiens Complete Proteome Set database (28 august 2013). Two missed cleavage sites were tolerated. The precursor mass tolerance was set to $5 \mathrm{ppm}$, the fragment mass tolerance to $0.05 \mathrm{Da}$. Carbamidomethylation of cysteins were allowed as static modification, methionine oxidation as dynamic modification.

For modeling the WebLogo (WebLogo 3, http://weblogo.threeplusone.com/create. cgi), all peptide identifications of the digest-immunoprecipitation triplicate were combined. Unspecific peptide binding to antibody and protein $\mathrm{G}$ was determined by analyses of immunoprecipitation experiments using (i) protein $\mathrm{G}$ bead and sample and (ii) protein G-beads and antibody without sample (measured in triplicates). Those peptides were considered as background and removed from the identification list. Peptide duplicates were removed. Identifications with at least two identical cterminal four-amino acid tags were further considered for modelling the epitope specificity. The weighting of amino acid presence was related to the total number of peptide spectrum matches (PSM, ProteomeDiscoverer 1.3, Thermo Scientific) across the triplicate. Parameters of the WebLogo software were kept default except for "units", which was set to "probability".

Dilution series in buffer. Isotopically-labeled peptides were diluted in digested fish gelatin, $50 \mathrm{mM}$ Tris-HCI, $150 \mathrm{mM} \mathrm{NaCl}$, and pH 7.4 (Blocking Reagent, Roche) while sequence-identical endogenous peptides were kept constant at a concentration of $10 \mathrm{nM}$. Starting from a concentration of $100 \mathrm{nM}$, the isotopically-labeled peptides were serially diluted eight times at a ratio of $1: 3$. According to the precipitation procedure, $10 \mu \mathrm{L}$ of each dilution step was incubated with $1 \mu \mathrm{g}$ anti-LPNK. For the [recommended] peptide sequence, an absolute peptide amount ranging from $1000 \mathrm{fmol}$ to $0.15 \mathrm{fmol}$ was provided for immunoprecipitation.

Dilution series in complex matrix. Isotopically-labeled peptides were diluted in hepatocyte extract. In an immunoprecipitation procedure, $1 \mu \mathrm{g}$ anti-LPNK was incubated with $10 \mu \mathrm{g}$ digested hepatocyte extract plus an isotopically-labeled peptide amount ranging from $1000 \mathrm{fmol}$ to $0.15 \mathrm{fmol}$.

Peptide quantification using LC-coupled targeted Single Ion Monitoring (LCtSIM). For quantification, the identical LC-setup was used as described in "Epitope analysis by LC-MS". Instead of a linear gradient, a step gradient starting at $15 \% \mathrm{~B}$ and ending after 8 min at 40\% B was chosen. On the QExactive Plus Orbitrap Mass
Spectrometer, a tSIM method was applied to quantify peptides at the parent ion level. The resolution was set to 70,000, the scan range was between 300 and $2000 \mathrm{~m} / \mathrm{z}$, and the AGC target was set at a value of 5e6. The injection time was adjusted to $120 \mathrm{~min}$. Loop count and MSX count were set to 2 respectively. An inclusion list registering the masses of endogenous and isotopically-labeled peptides was defined. For ensuring a high number of data points, the recording time for each signal pair was limited to $0.3 \mathrm{~min}$ prior and following the expected elution time. Data-dependent MS/MS was performed using a resolution of 17,500, an AGC-target of 2e5, and a maximum injection time of $120 \mathrm{~min}$. The collision energy was set to $30 \%$ and the underfill ratio to $0.6 \%$. For dynamic exclusion, a timeout of $2.0 \mathrm{sec}$ was chosen.

1. Method of the Year 2012. Nat. Methods 10, 1 (2013).

2. Gerber, S. A., Rush, J., Stemman, O., Kirschner, M. W. \& Gygi, S. P. Absolute quantification of proteins and phosphoproteins from cell lysates by tandem MS. Proc. Natl. Acad. Sci. USA 100, 6940-6945 (2003).

3. Picotti, P. et al. High-throughput generation of selected reaction-monitoring assays for proteins and proteomes. Nat. Methods 7, 43-46 (2010).

4. Gillette, M. A. \& Carr, S. A. Quantitative analysis of peptides and proteins in biomedicine by targeted mass spectrometry. Nat. Methods 10, 28-34 (2013).

5. Abbatiello, S. E., Mani, D. R., Keshishian, H. \& Carr, S. A. Automated detection of inaccurate and imprecise transitions in peptide quantification by multiple reaction monitoring mass spectrometry. Clin. Chem. 56, 291-305 (2010).

6. Anderson, N. L. et al. Mass spectrometric quantitation of peptides and proteins using Stable Isotope Standards and Capture by Anti-Peptide Antibodies (SISCAPA). J. Proteome Res. 3, 235-244 (2004).

7. Whiteaker, J. R. et al. A targeted proteomics-based pipeline for verification of biomarkers in plasma. Nat. Biotechnol. 29, 625-634 (2011).

8. Rissin, D. M. et al. Single-molecule enzyme-linked immunosorbent assay detects serum proteins at subfemtomolar concentrations. Nat. Biotechnol. 28, 595-599 (2010).

9. Uhlen, M. et al. A human protein atlas for normal and cancer tissues based on antibody proteomics. Mol. Cell. Proteomics 4, 1920-1932 (2005).

10. Haab, B. B. et al. A reagent resource to identify proteins and peptides of interest for the cancer community: a workshop report. Mol. Cell. Proteomics 5, 1996-2007 (2006).

11. Hoeppe, S. et al. Targeting peptide termini, a novel immunoaffinity approach to reduce complexity in mass spectrometric protein identification. Mol. Cell. Proteomics 10, M110 002857 (2011).

12. Planatscher, H. et al. Optimal selection of epitopes for TXP-immunoaffinity mass spectrometry. Algorithms Mol. Biol. 5, 28 (2010). 
13. Williamson, B. L. et al. Quantitative protein determination for CYP induction via LC-MS/MS. Proteomics 11, 33-41 (2011).

14. Kawakami, H. et al. Simultaneous absolute quantification of 11 cytochrome P450 isoforms in human liver microsomes by liquid chromatography tandem mass spectrometry with in silico target peptide selection. J. Pharm. Sci. 100, 341-352 (2011).

15. Sakamoto, A. et al. Reliability and robustness of simultaneous absolute quantification of drug transporters, cytochrome P450 enzymes, and Udpglucuronosyltransferases in human liver tissue by multiplexed MRM/selected reaction monitoring mode tandem mass spectrometry with nano-liquid chromatography. J. Pharm. Sci. 100, 4037-4043 (2011).

16. Jenkins, R. E. et al. Relative and absolute quantitative expression profiling of cytochromes $\mathrm{P} 450$ using isotope-coded affinity tags. Proteomics 6, 1934-1947 (2006).

17. Langenfeld, E., Zanger, U. M., Jung, K., Meyer, H. E. \& Marcus, K. Mass spectrometry-based absolute quantification of microsomal cytochrome P450 2D6 in human liver. Proteomics 9, 2313-2323 (2009).

18. Simicevic, J. et al. Absolute quantification of transcription factors during cellular differentiation using multiplexed targeted proteomics. Nat. Methods 10, 570-576 (2013).

19. Lewis, D. F. 57 varieties: the human cytochromes P450. Pharmacogenomics $\mathbf{5}$, 305-318 (2004).

20. Schaefer, O. et al. Absolute quantification and differential expression of drug transporters, cytochrome P450 enzymes, and UDP-glucuronosyltransferases in cultured primary human hepatocytes. Drug Metab. Dispos. 40, 93-103 (2012).

21. A Guidance for Industry - Bioanalytical method validation. US Food and Drug Administration (2001).

22. Feidt, D. M. et al. Profiling induction of cytochrome p450 enzyme activity by statins using a new liquid chromatography-tandem mass spectrometry cocktail assay in human hepatocytes. Drug Metab. Dispos. 38, 1589-1597 (2010).

23. Geick, A., Eichelbaum, M. \& Burk, O. Nuclear receptor response elements mediate induction of intestinal MDR1 by rifampin. J. Biol. Chem. 276, 14581-14587 (2001).

24. Burk, O. et al. Molecular mechanisms of polymorphic CYP3A7 expression in adult human liver and intestine. J. Biol. Chem. 277, 24280-24288 (2002).

25 . Chu, V. et al. In vitro and in vivo induction of cytochrome p450: a survey of the current practices and recommendations: a pharmaceutical research and manufacturers of america perspective. Drug Metab. Dispos. 37, 1339-1354 (2009).

26. Bucher, J. et al. A systems biology approach to dynamic modeling and intersubject variability of statin pharmacokinetics in human hepatocytes. BMC Syst. Biol. 5, 66 (2011).

27. Thasler, W. E. et al. Charitable State-Controlled Foundation Human Tissue and Cell Research: Ethic and Legal Aspects in the Supply of Surgically Removed Human Tissue For Research in the Academic and Commercial Sector in Germany. Cell Tissue Bank. 4, 49-56 (2003).
28. Nussler, A. K. et al. in Strategies in Regenerative Medicine. (ed. Santin, M.) (Springer, New York, 2008)

29. Cohen, S. A. \& Michaud, D. P. Synthesis of a fluorescent derivatizing reagent, 6 aminoquinolyl-N-hydroxysuccinimidyl carbamate, and its application for the analysis of hydrolysate amino acids via high-performance liquid chromatography. Anal. Biochem. 211, 279-287 (1993)

30. Plum, S. et al. Combined enrichment of neuromelanin granules and synaptosomes from human substantia nigra pars compacta tissue for proteomic analysis. J. Proteomics 94, 202-206 (2013).

31. Schaefer, H. et al. A peptide preconcentration approach for nano-highperformance liquid chromatography to diminish memory effects. Proteomics 4 2541-2544 (2004).

\section{Acknowledgments}

This work was funded by the German Federal Ministry of Education and Research (BMBF; grant FKZ 0315742 to M.F.T. and T.O.J. (Virtual Liver), grant FKZ 031A142 to F.W. and O.P. (GO-Bio), and grant FKZ 0313080 to A.S., M.R., A.K.N., T.S.W., W.E.T. and K.M. (HepatoSys network project)).

\section{Author contributions}

F.W., A.S., M.F.T., T.O.J., K.M. and O.P. conceived and planned the experiments. F.W., O.P., A.S., M.R. and B.S. performed experiments. A.K.N., W.E.T. and T.S.W. isolated hepatocytes. O.P., A.S., H.P., B.B., D.S. and F.W. performed data analysis. O.P. and K.M. prepared the manuscript. All authors discussed the results and commented on the paper.

\section{Additional information}

Supplementary information accompanies this paper at http://www.nature.com/ scientificreports

Competing financial interests: The authors declare no competing financial interests.

How to cite this article: Weiß, F. et al. Indirect protein quantification of drug-transforming enzymes using peptide group-specific immunoaffinity enrichment and mass spectrometry. Sci. Rep. 5, 8759; DOI:10.1038/srep08759 (2015)

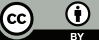

This work is licensed under a Creative Commons Attribution 4.0 International License. The images or other third party material in this article are included in the article's Creative Commons license, unless indicated otherwise in the credit line; if the material is not included under the Creative Commons license, users will need to obtain permission from the license holder in order to reproduce the material. To view a copy of this license, visit http://creativecommons.org/licenses/by/4.0/ 